\title{
Healthcare Costs and Workloss Burden of Patients with Chemotherapy-Associated Peripheral Neuropathy in Breast, Ovarian, Head and Neck, and Nonsmall Cell Lung Cancer
}

\author{
Crystal T. Pike, ${ }^{1}$ Howard G. Birnbaum, ${ }^{1}$ Catherine E. Muehlenbein, ${ }^{2}$ \\ Gerhardt M. Pohl, ${ }^{2}$ and Ronald B. Natale ${ }^{3}$ \\ ${ }^{1}$ Analysis Group, Inc., Boston, MA 02199, USA \\ ${ }^{2}$ Eli Lilly and Company, Indianapolis, IN 46285, USA \\ ${ }^{3}$ Cedars-Sinai Medical Center, Los Angeles, CA 90048, USA
}

Correspondence should be addressed to Crystal T. Pike, cpike@analysisgroup.com

Received 23 September 2011; Accepted 4 January 2012

Academic Editor: Vito Lorusso

Copyright (C 2012 Crystal T. Pike et al. This is an open access article distributed under the Creative Commons Attribution License, which permits unrestricted use, distribution, and reproduction in any medium, provided the original work is properly cited.

Objective. Chemotherapy-associated peripheral neuropathy (CAPN) is a painful side-effect of chemotherapy. This study assesses healthcare and workloss costs of CAPN patients with breast, ovarian, head/neck, or non-small cell lung cancer (NSCLC) from a third-party payor/employer perspective. Research Design and Methods. Patients with qualifying tumors, and claims for chemotherapy and services indicative of peripheral neuropathy (PN) within 9-months of chemotherapy (cases) were identified in a administrative claims database. Cases were matched $1: 1$ to controls with no PN-related claims based on demographics, diabetes history and propensity for having a diagnosis of PN during the study period (based on resource use and comorbidities in a 3month baseline period). Average all-cause healthcare costs, resource use and workloss burden were determined. Results. Average healthcare costs were $\$ 17,344$ higher for CAPN cases than their non-CAPN controls, with outpatient costs being the highest component (with cases having excess costs of \$8,092). On average, each CAPN case had 12 more outpatient visits than controls, and spent more days in the hospital. Workloss burden was higher for cases but not statistically different from controls. Conclusion. This study establishes that breast, ovarian, head/neck, or NSCLC patients with CAPN have significant excess healthcare costs and resource use.

\section{Introduction}

Chemotherapy-associated peripheral neuropathy (CAPN) is a neurological side effect of chemotherapy characterized by loss of sensation in the hands and feet, burning or tingling in limbs, and, in some cases, loss of hearing and blurred vision. The neuropathic symptoms are progressive and tend to increase as chemotherapy treatment proceeds. In addition, comorbid conditions may exacerbate the severity of CAPN [1]. For example, diabetes can lead to peripheral neuropathy, and diabetic patients with pre-existing nerve damage may be predisposed to more severe forms of CAPN [2-4].

The chemotherapy drugs most commonly associated with CAPN are taxanes (paclitaxel and docetaxel), vinca alkaloids (vincristine and vinorelbine), and platinums (cisplatin, carboplatin, and oxaliplatin). The incidence of CAPN varies by drug and dose and can range across products from 4$92 \%[1,2,5,6]$. For example, clinical trials of paclitaxel in breast cancer list incidence rates for severe CAPN between $2-33 \%$, with overall CAPN rates upwards of $60 \%[3,7,8]$. Currently, there are no standard treatments to prevent or mitigate CAPN, although several drug classes (e.g., tricyclic antidepressants, antiepileptic drugs, and adjuvant analgesics) have shown some activity in reducing neuropathic pain $[9,10]$.

Few data exist regarding the health outcomes of CAPN patients, CAPN's effects on chemotherapy treatment, and associated costs. However, Berger suggests neuropathies in general can lead to adverse outcomes and higher costs [11]. In particular, Berger found that patients with neuropathies had healthcare costs triple those of controls; however, the study 
did not examine the costs associated with chemotherapyrelated neuropathies specifically [11].

Calhoun conducted a pilot study on the medical and workloss costs associated with chemotherapy-induced toxicities in women with ovarian cancer [12]. Using survey data on 42 patients suffering from chemotherapy-induced neurotoxicities, the study found the medical costs directly attributable to CAPN were $\$ 688$ per episode but that indirect costs (patient and caregiver workloss and paid caregiver costs) were over $\$ 4,200$ per episode. This pilot study relied on patient recall of medical services used over 3-month intervals. In addition, the sample was limited to women with ovarian cancer and did not consider other cancer types.

To the authors' knowledge, no study has quantified the comprehensive health outcomes, medical costs, and workloss burden of CAPN patients with breast, ovarian, head/neck, or nonsmall cell lung cancer (NSCLC). The purpose of the current study is to assess health outcomes as well as the healthcare (i.e., medical and drug) and workloss cost burden of CAPN patients (cases) in these 4 tumor types from a thirdparty payor/employer perspective. The first objective is to compare the healthcare costs of CAPN cases with those of matched controls who have the same cancer but no CAPN. The second objective is to compare workloss costs in patients with and without CAPN. The third objective is to compare the healthcare costs of CAPN cases and non-CAPN controls who have comorbid diabetes. By examining these 4 tumor types, this study captures the use of the chemotherapeutic agents most commonly associated with CAPN.

\section{Methods}

2.1. Data. Data were obtained from a database of privately insured administrative claims records (Ingenix Employer Database) that included approximately 8 million beneficiaries from 40 large US-based companies (1999-2006). The companies have operations nationwide in a broad array of industries and job classifications. The database contains deidentified beneficiary information including demographics (e.g., age and gender), enrollment, and medical and pharmacy claims. Utilization measures include date of service, diagnoses, procedures, and actual payments to providers. Pharmaceutical drug claims include National Drug Code (NDC), fill date, days of supply, quantity, and actual payments. In addition, disability claims and employee wage information were available for employees in 23 companies.

2.2. Sample Selection. Three analytic samples consisting of CAPN cases and matched non-CAPN controls were used for this study. The main sample, consisting of cases and controls without diabetes, was used to evaluate the healthcare costs and resource use associated with CAPN patients. A sample of cases and controls with diabetes was used in the secondary analysis to evaluate the costs and resource use associated with diabetic CAPN patients. Finally, a subsample of employed cases and controls was used to assess the workloss burden associated with CAPN patients. Table 1(a) presents the sample selection.
2.2.1. Main CAPN Sample. Patients under age 65 were eligible for inclusion in the main sample if they had at least 1 claim with a diagnosis for 1 or more of the following cancers from 1999-2005: NSCLC, breast, ovarian, or head and neck. The cancer types were identified using ICD-9-CM codes (see Table 1(b)). To identify NSCLC from the overall lung cancer sample, patients receiving chemotherapy regimens characteristic of treatment for small cell lung cancer (SCLC) were excluded. SCLC treatment was defined in this study as doublet therapy with a platinum agent in combination with irinotecan, topotecan, or etoposide, or CCNU, melphalan, and VP-16 CAV regimen chemotherapy treatments. Of the patients with a claim for a qualifying tumor, only those with a procedure code indicating chemotherapy administration within 3 months of a claim for a qualifying tumor were selected. The date of first such chemotherapy administration was considered the index date. To ensure that the index event marked the start of a new line of chemotherapy for the tumor, patients were required to have at least 3 months of continuous eligibility prior to the index date with no claims for chemotherapy. Since no specific diagnosis code exists for CAPN, the authors developed an algorithm to define peripheral neuropathy (PN) using ICD-9-CM codes for related diagnoses and symptoms (see Table 1(b)). Any PN defined in the 9 months following the index date was assumed to be CAPN. Thus, patients were classified as CAPN if they had evidence of PN within 9 months of first chemotherapy treatment but had no evidence in the 3month baseline period. Finally, for this sample, patients with evidence of diabetes (i.e., a diagnosis of 250.x) from at least 3 months up to 12 months prior to the index date or during the 12 months following the index date were excluded.

2.2.2. Diabetic CAPN Sample. A sample of patients with 1 of the 4 tumor types, CAPN, and diabetes were selected for the secondary analysis. The patient selection criteria were the same as in the Main CAPN sample with the exception of the diabetes criteria. Specifically, diabetic CAPN patients were identified as those patients with a diagnosis of ICD-9-CM code 250.x anytime from at least 3 months up to 1 year prior to the index date or during the 12 months following the index date.

2.2.3. CAPN Employee Sample. A subsample of patients from the Main CAPN and Diabetic CAPN samples who were employees with disability data were selected for the workloss cost analysis. Note that this sample will not contain all employed persons from the main and diabetic CAPN samples but rather will only include the employees of the subset of companies with disability data available.

2.2.4. Study Period for All Samples. The study period encompassed the 12 months following the index date. Since the perspective adopted was that of the payor and the payor incurs no cost for patients who withdraw from coverage, no requirements were placed on the length of continuous eligibility following the index event. The baseline period for assessing patient history extended 3 months prior to the index date except for ascertaining the presence of diabetes. 
TABLE 1: Inclusion criteria and ICD-9-CM codes used for analytic samples.

(a) Inclusion criteria

\begin{tabular}{|c|c|c|}
\hline & Criteria & Number of patients \\
\hline 1 & Number of beneficiaries under 65 at eligibility start & $4,729,443$ \\
\hline 2 & $\begin{array}{l}>1 \text { breast, ovarian, head and neck, or nonsmall cell } \\
\text { lung cancer claim from 1999-2005 }\end{array}$ & 56,261 \\
\hline 3 & $\begin{array}{l}>1 \text { chemotherapy treatment within } 3 \text { months following } \\
\text { a breast, ovarian, head and neck, or nonsmall cell lung } \\
\text { cancer diagnosis }\end{array}$ & 14,142 \\
\hline 4 & $\begin{array}{l}>3 \text { months of continuous eligibility prior to the first } \\
\text { chemotherapy treatment for the qualifying tumor } \\
\text { during which no other chemotherapy treatment was } \\
\text { received }\end{array}$ & 11,009 \\
\hline 5 & $\begin{array}{l}>1 \text { diagnosis for peripheral neuropathy following first } \\
\text { chemotherapy treatment }\end{array}$ & 1,245 \\
\hline \multirow[t]{4}{*}{6} & $\begin{array}{l}\text { Peripheral neuropathy within } 9 \text { months of first } \\
\text { chemotherapy treatment }\end{array}$ & 525 \\
\hline & Main CAPN sample: no diabetes history & 454 \\
\hline & Diabetic CAPN sample & 71 \\
\hline & Employee CAPN subsample & 78 \\
\hline
\end{tabular}

(b) ICD-9-CM Diagnosis Codes Used for Identification of CAPN and Cancer

\begin{tabular}{lc}
\hline CAPN & ICD-9-CM code \\
\hline Polyneuropathy due to drugs & 357.6 \\
Disturbance of skin sensation & 782.0 \\
Inflammatory and toxic neuropathy & $357 . x$ \\
Toxic optic neuropathy & 377.34 \\
Reflex sympathetic dystrophy & 337.2 \\
Cervical root lesions & 353.2 \\
Lumbosacral root lesions & 353.4 \\
Other mononeuritis of unspecified site & 355.7 \\
Mononeuritis of unspecified site & 355.9 \\
Neuralgia, neuritis, or radiculitis & 729.2 \\
Brachial plexus lesions & 353.0 \\
\hline Cancer & ICD-9-CM code \\
\hline Nonsmall cell lung cancer & $162 . x$ \\
Breast cancer & $174 . x, 175 . x$ \\
Ovarian & $183 . x$ \\
Head and neck & $195.0,140 . x, 141 . x, 142 . x, 143 . x, 144 . x, 145 . x, 146 . x, 147 . x, 148 . x, 149 . x$ \\
\hline
\end{tabular}

In all cases, the patients were required to have a minimum of 3 months eligibility prior to the index date to qualify for the study.

2.3. Matching. Cases in the main CAPN sample and the diabetes CAPN sample were separately matched to controls selected from among the set of NSCLC, breast, ovarian, and head/neck cancer patients receiving chemotherapy who did not have a diagnosis for CAPN-related symptoms at any time in the claims history and were under age 65. Controls were matched to cases based on age, gender, employment status (employee versus nonemployee), cancer type, index date of chemotherapy, length of followup (controls were required to have postindex eligibility of at least as long as their matched case), and the estimated likelihood of developing CAPN. Each case was matched $1: 1$ to a control using an optimal matching algorithm [13]. Likelihood of developing CAPN was derived as a propensity score from a logistic regression model based on resource use and comorbidities during the baseline period. Specifically, the model included age, sex, Charlson Comorbidity Index (CCI) [14], number of inpatient, primary care, oncology, neurology, and other physician visits, and binary variables indicating whether patient went to the 
emergency room or had depression or uncomplicated hypertension.

The time period over which controls contributed to the outcomes measures was truncated at the end of the observation period for their matching case (i.e., the earlier of either 12 months or when cases dropped from the database).

2.4. Measures. Healthcare costs were stratified into 3 mutually exclusive groups: chemotherapy costs, drug costs, and medical costs. Costs were computed as the paid (reimbursed) amount by the insurer to the health-care provider and were annualized to 2006 U.S. Dollars using the Consumer Price Index for medical care. Chemotherapy costs included medical claims with a chemotherapy procedure code and pharmacy claims for oral chemotherapy agents, identified by NDCs. Drug costs included all pharmaceutical claims other than chemotherapy claims. Medical costs included claims for inpatient, emergency department (ED), and outpatient/other care. Costs included all claims associated with any service provided to the case/control during the study period regardless of diagnosis, procedure, or drug. The subset of pharmaceutical and medical costs that could be directly attributed to CAPN was estimated as follows: CAPN-related drug costs included those for drugs potentially used for CAPN (i.e., amitriptyline, gabapentin, amifostine, glutamine, tricyclic antidepressants, anti-epileptics, NSAIDs, and opioids) and CAPN-related medical costs included those from claims with a primary or secondary diagnosis of a CAPN-related symptom (see Table 1(b)).

Healthcare resource use consisted of hospitalizations, emergency department (ED), and outpatient/other services (reported by type of visit). The resource use components were defined using provider specialty codes and/or place of service codes on the claims. All claims during the study period were included in assessing resource use, regardless of the underlying reason for a visit. In addition, the proportion of patients using CAPN-related drugs, and specific chemotherapy agents were measured.

Workloss days and costs consisted of disability and medically related absenteeism. Workloss costs during the 12month study period included actual employer payments for disability days plus imputed costs for medically related absenteeism. Medically related absenteeism costs were imputed by multiplying the number of days with medical services resource use by the employee's wage: each hospitalization day accounted for a full day of workloss, and outpatient visits accounted for half a day of workloss. As with healthcare costs, total workloss costs were not limited to only those related to a particular condition.

Patient characteristics included demographics, employment status (employee versus nonemployee), cancer type, cancer stage (metastatic versus not metastatic), and comorbidities identified using claims during baseline period and the 12-month study period. Metastatic cancer was defined using claims with diagnoses for metastases (ICD-9-CM codes 196.0-199.1). The CCI was calculated from the claims data and individual physical comorbidities included in the index which were also identified [14].
2.5. Statistical Analyses. Baseline characteristics (demographics, comorbidities, resource use rates) were summarized as proportions of the sample with the characteristic. Continuous measures (e.g., healthcare costs, workloss costs, resource use amount) were summarized by mean and standard deviation. Comparisons of matched pairs of categorical variables used McNemar tests. Comparisons of the differences in continuous measures between cases and controls used paired $t$-tests. Excess costs of CAPN cases compared with controls (i.e., costs of CAPN cases minus those of controls) were compared between diabetic and nondiabetic patients using a 2-sample $t$-test. All analyses were conducted using SAS version 9.1 (SAS Institute Inc., Cary, NC). Pvalues less than or equal to 0.05 were considered statistically significant.

\section{Results}

The main study sample for the healthcare cost and resource use analysis contained 454 cases and controls without diabetes (see Table 1(a)). The diabetes sample for the secondary analysis contained 71 diabetic cases and controls. The employee subsample for the workloss cost and resource use analysis contained 78 cases and controls.

3.1. Baseline Characteristics. Tables 2(A) and 3(A) show the baseline comparison of CAPN cases and their matched nonCAPN controls within the main sample. Cases and controls were balanced in terms of types of cancer and CCI. However, CAPN cases had more congestive heart failure (4\% versus $2 \%, P=0.0412)$ and uncomplicated hypertension $(17 \%$ versus $11 \%, P=0.0164)$, whereas the control group had a higher rate of complicated hypertension (3\% versus $1 \%, P=$ $0.0290)$. Resource use was generally the same between cases and controls; however, there were more cases with neurology specialist visits than controls (5\% versus $2 \%, P=0.0482)$. The number of neurology visits and neurology costs were not significantly different.

3.2. Study Period Descriptive Characteristics, Healthcare Resource Use, and Costs. CAPN cases had significantly higher rates and counts of comorbidities and resource use during the study period compared with matched non-CAPN controls (Tables 4(A) and 5(A)). Significantly more cases had fibromyalgia, obesity, and uncomplicated hypertension during the study period than their matched controls. Cases also had a higher CCI during the study period (4.7 versus $4.1, P<0.0001)$. There were no differences in the classes of chemotherapy agents used by cases and controls. The most common chemotherapeutic agents used by cases and controls were taxanes $(33 \%, 28 \%)$ and platinums $(20 \%$, $18 \%)$. Significantly more cases used a CAPN-related drug than controls $(72 \%$ versus $56 \%, P<0.0001)$. Cases had substantially higher rates and amounts of use of both inpatient and outpatient visits during the study period. More cases were hospitalized at least once compared with controls (51\% versus $37 \%, P<0.0001$ ). CAPN cases had significantly higher rates and amounts of use for all outpatient components except for primary care visits and lab/pathology. More 
TABLE 2: Three-month baseline demographics and comorbidities of CAPN cases and non-CAPN controls.

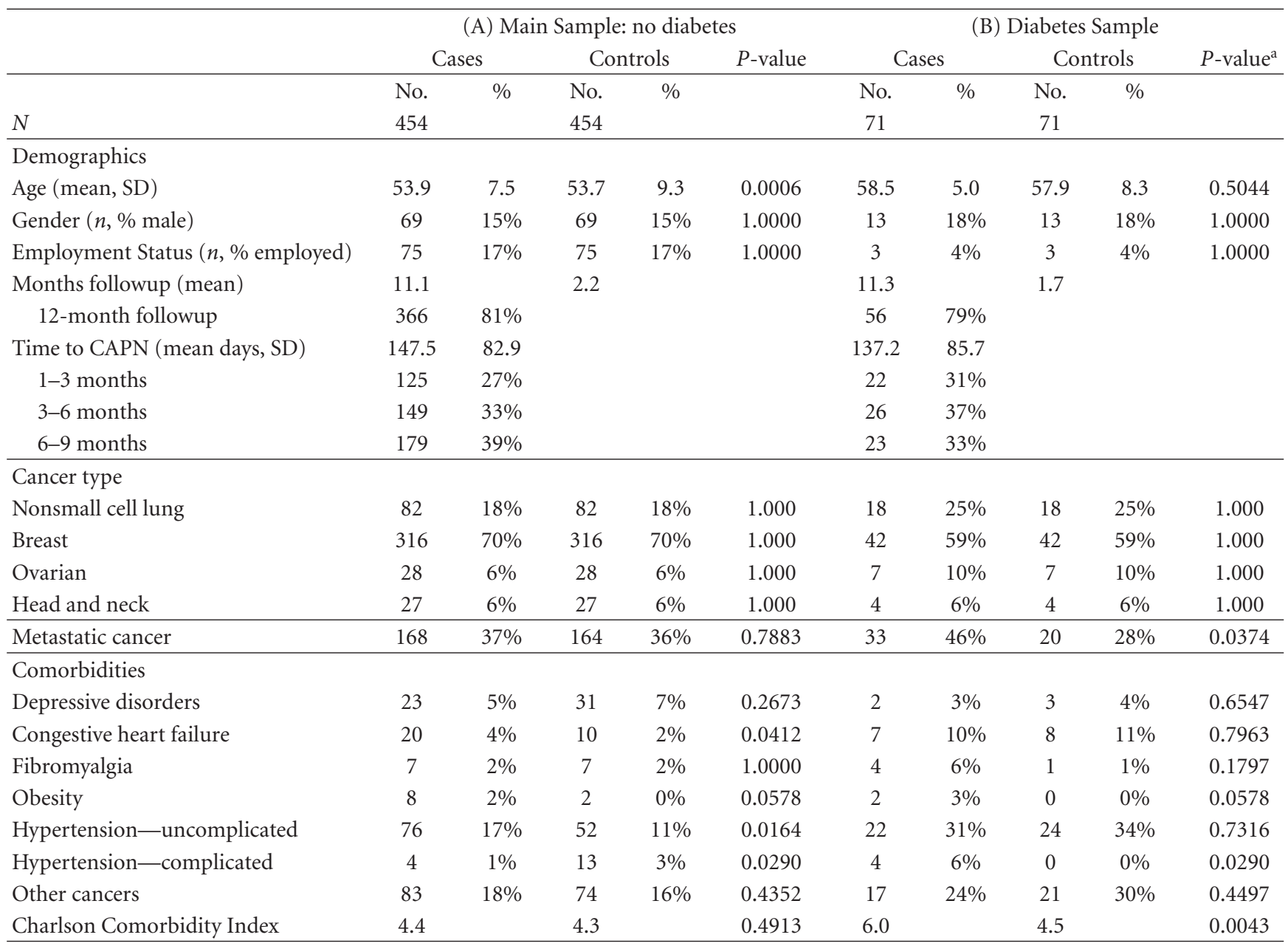

CAPN indicates chemotherapy-associated peripheral neuropathy; SD, standard deviation.

${ }^{a} P$-values are determined using McNemar tests for proportions and paired $t$-tests for continuous measures.

cases saw a neurologist than did controls (29\% versus $6 \%$, $P<0.0001)$.

Table 6(A) shows the healthcare cost comparison for cases and controls. For cases, mean annual per capita healthcare costs were $\$ 69,950$ versus $\$ 52,606$ per control, with an excess annual per patient cost of $\$ 17,344(P<0.0001)$. Mean excess annual per patient healthcare costs for cases versus controls were $\$ 36,660$ for head and neck cancer, $\$ 18,790$ for nonsmall cell lung cancer, $\$ 16,940$ for breast cancer, and $\$ 5,140$ for ovarian and all were statistically significant (data not shown). Cases had significantly higher component costs compared with controls. Outpatient costs were the highest component for both cases and controls. However, the excess costs of outpatient and inpatient components were similar with cases having excess annual outpatient costs of $\$ 8,092$ per patient $(P<0.0001)$ and excess annual inpatient costs of $\$ 7,552$ per patient $(P<0.0001)$. Annual oncologyrelated costs (i.e., chemotherapy and oncologist specialist costs) were $\$ 22,453$ for cases compared with $\$ 19,362$ for controls, with the majority of costs being chemotherapy costs (\$16,984 and $\$ 16,169$ for cases and controls, resp., $P=$
0.5744). CAPN-related drug and medical costs accounted for approximately $2 \%$ of total healthcare costs.

3.3. Workloss and Costs. There were no statistically significant differences in workloss measures between the subset of 78 employees with CAPN and their matched non-CAPN controls (Table 7). More cases had disability claims than controls ( $35 \%$ versus $26 \%$, resp.). Cases also had almost twice as many disability days as their matched controls (37.4 versus 20.4 days, resp.). While almost all cases and controls had at least 1 medically related absenteeism day (95\% and 97\%, resp.), cases missed 6 additional days over the 12-month study period compared with controls.

Average annual workloss costs were approximately 25\% higher for cases than controls ( $\$ 11,298$ versus $\$ 9,043$, resp.) with a $\$ 2,255$ annual per patient difference (Table 7). Cases had both higher disability and higher medically-related absenteeism costs than controls $(\$ 4,970$ versus $\$ 3,356$ for disability and $\$ 6,329$ versus $\$ 5,687$ for medically-related absenteeism). However, workloss cost differences were not statistically significant. 
TABLE 3: Three-month per-capita baseline resource use and healthcare costs of CAPN cases and non-CAPN controls.

\begin{tabular}{|c|c|c|c|c|c|c|c|c|c|c|}
\hline & \multicolumn{5}{|c|}{ (A) Main Sample: no diabetes } & \multicolumn{5}{|c|}{ (B) Diabetes Sample } \\
\hline & \multicolumn{2}{|c|}{ Cases } & \multicolumn{2}{|c|}{ Controls } & \multirow[t]{3}{*}{$P$-value ${ }^{\mathrm{a}}$} & \multicolumn{2}{|c|}{ Cases } & \multicolumn{2}{|c|}{ Controls } & \multirow[t]{3}{*}{$P$-value ${ }^{\mathrm{a}}$} \\
\hline & No. & $\%$ & No. & $\%$ & & No. & $\%$ & No. & $\%$ & \\
\hline$N$ & 454 & & 454 & & & 71 & & 71 & & \\
\hline \multicolumn{11}{|l|}{ Resource use rate } \\
\hline Hospitalizations & 204 & $45 \%$ & 193 & $43 \%$ & 0.4692 & 43 & $61 \%$ & 33 & $46 \%$ & 0.1138 \\
\hline ED visits & 75 & $17 \%$ & 68 & $15 \%$ & 0.5139 & 21 & $30 \%$ & 16 & $23 \%$ & 0.3980 \\
\hline Outpatient visits & 444 & $98 \%$ & 445 & $98 \%$ & 0.8185 & 71 & $100 \%$ & 70 & $99 \%$ & 0.8185 \\
\hline Oncology & 188 & $41 \%$ & 174 & $38 \%$ & 0.3408 & 26 & $37 \%$ & 30 & $42 \%$ & 0.4795 \\
\hline Neurology & 22 & $5 \%$ & 11 & $2 \%$ & 0.0482 & 5 & $7 \%$ & 1 & $1 \%$ & 0.1025 \\
\hline Resource use amount & Mean & $(\mathrm{SD})$ & Mean & $(\mathrm{SD})$ & $P$-value ${ }^{\mathrm{a}}$ & Mean & $(\mathrm{SD})$ & Mean & $(\mathrm{SD})$ & $P$-value ${ }^{\mathrm{a}}$ \\
\hline Hospitalizations & 1.96 & $(5.53)$ & 1.48 & $(3.21)$ & 0.0956 & 3.48 & $(5.02)$ & 4.10 & $(9.75)$ & 0.5702 \\
\hline ED visits & 0.24 & $(0.70)$ & 0.21 & $(0.58)$ & 0.3550 & 0.41 & $(0.73)$ & 0.35 & $(0.76)$ & 0.6310 \\
\hline Outpatient visits & 11.37 & $(6.80)$ & 11.25 & $(7.40)$ & 0.7449 & 12.80 & $(6.08)$ & 11.28 & $(6.76)$ & 0.1420 \\
\hline Oncology & 1.40 & $(3.06)$ & 1.33 & $(3.47)$ & 0.6673 & 1.38 & $(3.10)$ & 1.44 & $(2.97)$ & 0.9040 \\
\hline Neurology & 0.06 & $(0.31)$ & 0.03 & $(0.22)$ & 0.0848 & 0.07 & $(0.26)$ & 0.01 & $(0.12)$ & 0.1029 \\
\hline Total costs & $\$ 17,797$ & $(\$ 22,255)$ & $\$ 17,180$ & $(\$ 25,240)$ & 0.6698 & $\$ 22,308$ & $(\$ 24,970)$ & $\$ 21,061$ & $(\$ 22,748)$ & 0.6898 \\
\hline Drug costs & $\$ 433$ & $(\$ 857)$ & $\$ 467$ & $(\$ 1,148)$ & 0.6064 & $\$ 800$ & $(\$ 868)$ & $\$ 646$ & $(\$ 824)$ & 0.2761 \\
\hline Medical costs & $\$ 17,363$ & $(\$ 22,185)$ & $\$ 16,712$ & $(\$ 25,140)$ & 0.6527 & $\$ 21,508$ & $(\$ 24,871)$ & $\$ 20,415$ & $(\$ 22,636)$ & 0.7268 \\
\hline Inpatient & $\$ 6,496$ & $(\$ 19,301)$ & $\$ 5,928$ & $(\$ 21,811)$ & 0.6632 & $\$ 11,220$ & $(\$ 22,256)$ & $\$ 9,382$ & $(\$ 21,412)$ & 0.4845 \\
\hline $\mathrm{ED}$ & $\$ 127$ & $(\$ 826)$ & $\$ 102$ & $(\$ 576)$ & 0.6043 & $\$ 125$ & $(\$ 290)$ & $\$ 122$ & $(\$ 340)$ & 0.9490 \\
\hline Outpatient & $\$ 10,336$ & $(\$ 10,186)$ & $\$ 10,328$ & $(\$ 10,282)$ & 0.9900 & $\$ 9,725$ & $(\$ 8,672)$ & $\$ 10,358$ & $(\$ 9,840)$ & 0.6935 \\
\hline Oncology & $\$ 438$ & $(\$ 1,329)$ & $\$ 439$ & $(\$ 1,796)$ & 0.9841 & $\$ 379$ & $(\$ 996)$ & $\$ 431$ & $(\$ 1,452)$ & 0.7989 \\
\hline Neurology & $\$ 10$ & $(\$ 61)$ & $\$ 9$ & $(\$ 108)$ & 0.8812 & $\$ 24$ & $(\$ 163)$ & $\$ 1$ & $(\$ 7)$ & 0.2435 \\
\hline
\end{tabular}

CAPN indicates chemotherapy-associated peripheral neuropathy; SD, standard deviation; ED, emergency department.

${ }^{a} P$-values are determined using McNemar tests for proportions and paired $t$-tests for continuous measures.

TABLE 4: Chemotherapy agents and comorbidities of CAPN cases and non-CAPN controls during the 12-month study period.

\begin{tabular}{|c|c|c|c|c|c|c|c|c|c|c|}
\hline & \multicolumn{5}{|c|}{ (A) Main Sample: no diabetes } & \multicolumn{5}{|c|}{ (B) Diabetes Sample } \\
\hline & \multicolumn{2}{|c|}{ Cases } & \multicolumn{2}{|c|}{ Controls } & \multirow[t]{2}{*}{$P$-value ${ }^{\mathrm{a}}$} & \multicolumn{2}{|c|}{ Cases } & \multicolumn{2}{|c|}{ Controls } & \multirow[t]{3}{*}{$P$-value } \\
\hline & No. & $\%$ & No. & $\%$ & & No. & $\%$ & No. & $\%$ & \\
\hline$N$ & 454 & & 454 & & & 71 & & 71 & & \\
\hline Metastatic cancer & 171 & $38 \%$ & 136 & $30 \%$ & 0.0097 & 30 & $42 \%$ & 22 & $31 \%$ & 0.1306 \\
\hline \multicolumn{11}{|c|}{ Classes of select chemotherapy agents used } \\
\hline Taxanes & 148 & $33 \%$ & 125 & $28 \%$ & 0.0978 & 30 & $42 \%$ & 17 & $24 \%$ & 0.0236 \\
\hline Vinca Alkaloids & 18 & $4 \%$ & 14 & $3 \%$ & 0.4497 & 3 & $4 \%$ & 2 & $3 \%$ & 0.6547 \\
\hline Platinums & 90 & $20 \%$ & 80 & $18 \%$ & 0.4014 & 17 & $24 \%$ & 20 & $28 \%$ & 0.5775 \\
\hline \multicolumn{11}{|l|}{ Comorbidities } \\
\hline Depressive disorders & 68 & $15 \%$ & 50 & $11 \%$ & 0.0804 & 8 & $11 \%$ & 3 & $4 \%$ & 0.1317 \\
\hline Congestive heart failure & 23 & $5 \%$ & 20 & $4 \%$ & 0.6219 & 12 & $17 \%$ & 12 & $17 \%$ & 1.0000 \\
\hline Fibromyalgia & 37 & $8 \%$ & 8 & $2 \%$ & $<.0001$ & 8 & $11 \%$ & 1 & $1 \%$ & 0.0196 \\
\hline Obesity & 9 & $2 \%$ & 2 & $0 \%$ & $<.0001$ & 1 & $1 \%$ & 0 & $0 \%$ & 0.2568 \\
\hline Hypertension-uncomplicated & 114 & $25 \%$ & 83 & $18 \%$ & 0.0134 & 33 & $46 \%$ & 35 & $49 \%$ & 0.7576 \\
\hline Hypertension—complicated & 13 & $3 \%$ & 13 & $3 \%$ & 1.0000 & 5 & $7 \%$ & 1 & $1 \%$ & 0.1025 \\
\hline Other cancers ${ }^{\mathrm{b}}$ & 124 & $27 \%$ & 101 & $22 \%$ & 0.0838 & 20 & $28 \%$ & 19 & $27 \%$ & 0.8415 \\
\hline Charlson Comorbidity Index & 4.7 & & 4.1 & & $<.0001$ & 7.0 & & 5.6 & & 0.0052 \\
\hline
\end{tabular}

CAPN indicates chemotherapy-associated peripheral neuropathy.

${ }^{a} P$-values are determined using McNemar tests for proportions and paired $t$-tests for continuous measures.

b Other cancers include all cancers other than head and neck, breast, non-small cell lung, and ovarian. 
TABLE 5: Resource use of CAPN cases and non-CAPN controls during the 12-month study period.

\begin{tabular}{|c|c|c|c|c|c|c|c|c|c|c|}
\hline & \multicolumn{5}{|c|}{ (A) Main Sample: no diabetes } & \multicolumn{5}{|c|}{ (B) Diabetes Sample } \\
\hline & \multicolumn{2}{|c|}{ Cases } & \multicolumn{2}{|c|}{ Controls } & \multirow[t]{2}{*}{$P$-value ${ }^{\mathrm{a}}$} & \multicolumn{2}{|c|}{ Cases } & \multicolumn{2}{|c|}{ Controls } & \multirow[t]{2}{*}{$P$-value } \\
\hline & No. & $\%$ & No. & $\%$ & & No. & $\%$ & No. & $\%$ & \\
\hline$N$ & 454 & & 454 & & & 71 & & 71 & & \\
\hline \multicolumn{11}{|l|}{ Resource use rate } \\
\hline \multicolumn{11}{|l|}{ Medical } \\
\hline Hospitalizations & 231 & $51 \%$ & 166 & $37 \%$ & $<.0001$ & 48 & $68 \%$ & 32 & $45 \%$ & 0.0136 \\
\hline ED visits & 213 & $47 \%$ & 170 & $37 \%$ & 0.0037 & 42 & $59 \%$ & 27 & $38 \%$ & 0.0222 \\
\hline Outpatient visits & 453 & $100 \%$ & 447 & $98 \%$ & 0.0339 & 71 & $100 \%$ & 70 & $99 \%$ & 0.0339 \\
\hline Oncology & 288 & $63 \%$ & 250 & $55 \%$ & 0.0075 & 43 & $61 \%$ & 39 & $55 \%$ & 0.5050 \\
\hline Neurology & 133 & $29 \%$ & 29 & $6 \%$ & $<.0001$ & 30 & $42 \%$ & 5 & $7 \%$ & $<.0001$ \\
\hline Primary care & 354 & $78 \%$ & 345 & $76 \%$ & 0.4726 & 59 & $83 \%$ & 60 & $85 \%$ & 0.8185 \\
\hline Other physician & 444 & $98 \%$ & 428 & $94 \%$ & 0.0035 & 71 & $100 \%$ & 66 & $93 \%$ & 0.0035 \\
\hline Lab/pathology & 181 & $40 \%$ & 160 & $35 \%$ & 0.1540 & 21 & $30 \%$ & 22 & $31 \%$ & 0.8694 \\
\hline Other outpatient & 400 & $88 \%$ & 369 & $81 \%$ & 0.0030 & 68 & $96 \%$ & 61 & $86 \%$ & 0.0522 \\
\hline \multicolumn{11}{|l|}{ Prescription drug use } \\
\hline At least 1 CAPN-related drug & 329 & $72 \%$ & 256 & $56 \%$ & $<.0001$ & 66 & $93 \%$ & 46 & $65 \%$ & 0.0003 \\
\hline Resource use amount & Mean & $(\mathrm{SD})$ & Mean & $(\mathrm{SD})$ & $P$-value ${ }^{\mathrm{a}}$ & Mean & $(\mathrm{SD})$ & Mean & $(\mathrm{SD})$ & $P$-value \\
\hline \multicolumn{11}{|l|}{ Medical } \\
\hline Hospitalizations & 5.6 & $(11.69)$ & 3.2 & $(7.77)$ & 0.0001 & 9.1 & $(14.38)$ & 6.2 & $(13.44)$ & 0.2195 \\
\hline ED visits & 1.1 & $(2.80)$ & 0.6 & $(1.28)$ & 0.0022 & 1.6 & $(1.98)$ & 0.8 & $(1.35)$ & 0.0064 \\
\hline Outpatient visits & 51.3 & $(29.57)$ & 39.8 & $(26.81)$ & $<.0001$ & 56.9 & $(27.02)$ & 38.5 & $(25.10)$ & $<.0001$ \\
\hline Oncology & 12.7 & $(18.92)$ & 9.2 & $(15.55)$ & 0.0021 & 12.6 & $(16.97)$ & 7.5 & $(10.62)$ & 0.0322 \\
\hline Neurology & 0.6 & $(1.29)$ & 0.1 & $(0.51)$ & $<.0001$ & 1.1 & $(1.87)$ & 0.1 & $(0.56)$ & 0.0002 \\
\hline Primary care & 6.9 & (12.65) & 5.0 & $(8.74)$ & 0.0085 & 7.4 & $(10.25)$ & 5.7 & $(5.64)$ & 0.2454 \\
\hline Other physician & 22.1 & $(21.22)$ & 17.2 & $(18.82)$ & 0.0001 & 25.9 & $(20.63)$ & 18.6 & $(18.82)$ & 0.0217 \\
\hline Lab/pathology & 1.1 & $(2.62)$ & 0.9 & $(2.49)$ & 0.2127 & 0.8 & $(3.59)$ & 1.1 & $(3.93)$ & 0.6594 \\
\hline Other outpatient & 13.6 & (16.47) & 11.4 & (15.97) & 0.0349 & 15.5 & (15.37) & 11.0 & (16.56) & 0.0905 \\
\hline
\end{tabular}

CAPN indicates chemotherapy-associated peripheral neuropathy; SD, standard deviation; ED, emergency department.

${ }^{a} P$-values are determined using McNemar tests for proportions and paired $t$-tests for continuous measures.

3.4. Secondary Analysis of CAPN and Diabetes. Tables 2(B) and $3(\mathrm{~B})$ show the baseline comparison of diabetic cases and their matched diabetic controls. Cases had more metastatic disease ( $46 \%$ versus $28 \%, P=0.0374)$, complicated hypertension ( $6 \%$ versus $0 \%, P=0.0290)$, and a higher CCI than controls (6.0 versus $4.5, P=0.0043)$. Resource use was generally well balanced; however, cases did have more primary care visits than controls (2.75 versus $1.75, P=$ $0.0136)$. There were no statistically significant differences in baseline costs.

Cases had significantly higher rates and counts of resource use during the study period compared with controls (see Tables 4(B) and 5(B)). More cases than controls used taxanes $(42 \%$ versus $24 \%, P=0.0236$ ). More cases were hospitalized compared with controls $(68 \%$ versus $45 \%, P=$ $0.0136)$ though the days per capita were not statistically different (9.1 versus 6.2 for cases and controls, resp., $P=$ $0.2195)$. Six times as many cases had a neurology specialist visit during the study period compared with controls ( $42 \%$ versus $7 \%, P<0.0001$ ). Cases also had 18.4 more outpatient visits during the study period on average than the controls
$(P<0.0001)$. CAPN-related drugs were used by $93 \%$ of cases compared with $65 \%$ of controls $(P=0.0003)$.

For cases, annual per capita healthcare costs were $\$ 76,555$ versus $\$ 54,816$ per control, with an excess annual per patient cost of $\$ 21,739(P=0.0273$, Table 6(B)). Annual oncology-related costs were $\$ 25,181$ for cases compared with $\$ 15,377$ for controls, with the majority of these costs being chemotherapy costs $(\$ 20,990$ and $\$ 13,033$ for cases and controls, resp., $P=0.0670$ ). The diabetic case's annual per capita excess costs were higher than the control's costs $(\$ 21,739$ versus $\$ 17,344)$, however, this difference was not statistically significant.

\section{Discussion}

This study is the first to use claims data to estimate the excess costs of CAPN patients in breast, nonsmall cell lung, ovarian, and head and neck cancer over a matched sample of cancer patients without CAPN. This study also included a secondary analysis to examine the excess costs of CAPN patients among patients with comorbid diabetes. 
TABLE 6: Per capita healthcare costs for CAPN cases and non-CAPN controls during the 12-month study period.

\begin{tabular}{|c|c|c|c|c|c|c|c|c|c|c|}
\hline & \multicolumn{5}{|c|}{ (A) Main Sample: no diabetes } & \multicolumn{5}{|c|}{ (B) Diabetes Sample } \\
\hline & \multicolumn{2}{|c|}{ Cases } & \multicolumn{2}{|c|}{ Controls } & \multirow[t]{2}{*}{$P$-value ${ }^{\mathrm{a}}$} & \multicolumn{2}{|c|}{ Cases } & \multicolumn{2}{|c|}{ Controls } & \multirow[t]{2}{*}{$P$-value } \\
\hline & mean & $(\mathrm{SD})$ & mean & $(\mathrm{SD})$ & & mean & $(\mathrm{SD})$ & mean & $(\mathrm{SD})$ & \\
\hline$N$ & 454 & & 454 & & & 71 & & 71 & & \\
\hline Total healthcare costs & $\$ 69,950$ & $(\$ 66,913)$ & $\$ 52,606$ & $(\$ 55,554)$ & $<.0001$ & $\$ 76,555$ & $(\$ 63,379)$ & $\$ 54,816$ & $(\$ 68,115)$ & 0.0273 \\
\hline Chemotherapy costs & $\$ 16,984$ & $(\$ 21,248)$ & $\$ 16,169$ & $(\$ 27,055)$ & 0.5744 & $\$ 20,990$ & $(\$ 31,501)$ & $\$ 13,033$ & $(\$ 19,725)$ & 0.0670 \\
\hline Drug costs & $\$ 3,744$ & $(\$ 5,333)$ & $\$ 3,071$ & $(\$ 4,927)$ & 0.0419 & $\$ 6,017$ & $(\$ 7,461)$ & $\$ 4,223$ & $(\$ 6,100)$ & 0.1163 \\
\hline CAPN-related drugs & $\$ 595$ & $(\$ 1,590)$ & $\$ 328$ & $(\$ 1,041)$ & 0.0016 & $\$ 718$ & $(\$ 1,401)$ & $\$ 371$ & $(\$ 782)$ & 0.0832 \\
\hline Medical costs & $\$ 49,223$ & $(\$ 56,500)$ & $\$ 33,366$ & $(\$ 36,931)$ & $<.0001$ & $\$ 49,548$ & $(\$ 46,428)$ & $\$ 37,561$ & $(\$ 57,723)$ & 0.1614 \\
\hline Inpatient & $\$ 14,050$ & $(\$ 35,793)$ & $\$ 6,498$ & $(\$ 15,558)$ & $<.0001$ & $\$ 19,181$ & $(\$ 38,190)$ & $\$ 15,148$ & $(\$ 44,098)$ & 0.5637 \\
\hline $\mathrm{ED}$ & $\$ 474$ & $(\$ 1,093)$ & $\$ 263$ & $(\$ 730)$ & 0.0005 & $\$ 798$ & $(\$ 1,817)$ & $\$ 243$ & $(\$ 535)$ & 0.0172 \\
\hline Outpatient & $\$ 34,698$ & $(\$ 36,712)$ & $\$ 26,606$ & $(\$ 29,913)$ & $<.0001$ & $\$ 29,569$ & $(\$ 21,339)$ & $\$ 22,170$ & $(\$ 29,244)$ & 0.0921 \\
\hline Oncology & $\$ 5,469$ & $(\$ 15,923)$ & $\$ 3,193$ & $(\$ 8,478)$ & 0.0072 & $\$ 4,191$ & $(\$ 6,950)$ & $\$ 2,344$ & $(\$ 4,729)$ & 0.0554 \\
\hline Neurology & $\$ 129$ & $(\$ 319)$ & $\$ 46$ & $(\$ 433)$ & 0.0011 & $\$ 326$ & $(\$ 843)$ & $\$ 44$ & $(\$ 278)$ & 0.0103 \\
\hline Primary care & $\$ 7,772$ & $(\$ 12,454)$ & $\$ 6,543$ & $(\$ 9,920)$ & 0.0979 & $\$ 6,024$ & $(\$ 7,341)$ & $\$ 4,472$ & $(\$ 5,965)$ & 0.1252 \\
\hline Other physician & $\$ 7,437$ & $(\$ 13,782)$ & $\$ 6,804$ & $(\$ 17,452)$ & 0.5247 & $\$ 6,235$ & $(\$ 11,447)$ & $\$ 5,150$ & $(\$ 16,025)$ & 0.6502 \\
\hline Lab/pathology & $\$ 414$ & $(\$ 1,825)$ & $\$ 289$ & $(\$ 1,490)$ & 0.2632 & $\$ 485$ & $(\$ 2,027)$ & $\$ 110$ & $(\$ 193)$ & 0.1237 \\
\hline Other outpatient & $\$ 13,476$ & $(\$ 24,509)$ & $\$ 9,731$ & $(\$ 18,345)$ & 0.0064 & $\$ 12,307$ & $(\$ 16,297)$ & $\$ 10,049$ & $(\$ 20,812)$ & 0.4798 \\
\hline CAPN-related medical costs & $\$ 725$ & $(\$ 2,005)$ & & & & $\$ 491$ & $(\$ 762)$ & & & \\
\hline
\end{tabular}

CAPN indicates chemotherapy-associated peripheral neuropathy; SD, standard deviation; ED, emergency department.

${ }^{a} P$-values are determined using McNemar tests for proportions and paired $t$-tests for continuous measures.

TABle 7: Per capita workloss days and costs during the 12-month study period.

\begin{tabular}{|c|c|c|c|c|c|}
\hline & \multicolumn{5}{|c|}{ Employee subsample } \\
\hline & \multicolumn{2}{|c|}{ Cases } & \multicolumn{2}{|c|}{ Controls } & \multirow[t]{3}{*}{$P$-value } \\
\hline & No. & $\%$ & No. & $\%$ & \\
\hline$N$ & 78 & & 78 & & \\
\hline \multicolumn{6}{|l|}{ Workloss rate ${ }^{b}$} \\
\hline $\begin{array}{l}\text { Medically } \\
\text { related } \\
\text { absenteeism }\end{array}$ & 74 & $95 \%$ & 76 & $97 \%$ & 0.4142 \\
\hline Disability & 27 & $35 \%$ & 20 & $26 \%$ & 0.2367 \\
\hline Workloss days & mean & $(\mathrm{SD})$ & Mean & $(\mathrm{SD})$ & $P$-value ${ }^{\mathrm{a}}$ \\
\hline $\begin{array}{l}\text { Medically } \\
\text { related } \\
\text { absenteeism }\end{array}$ & 31.5 & $(28.1)$ & 25.3 & $(18.2)$ & 0.0573 \\
\hline Disability & 37.4 & $(75.4)$ & 20.4 & $(50.8)$ & 0.1048 \\
\hline Workloss costs & mean & $(\mathrm{SD})$ & mean & $(\mathrm{SD})$ & $P$-value ${ }^{\mathrm{a}}$ \\
\hline $\begin{array}{l}\text { Total employer } \\
\text { costs }\end{array}$ & $\$ 11,298$ & $(\$ 11,830)$ & $\$ 9,043$ & $(\$ 12,416)$ & 0.2161 \\
\hline $\begin{array}{l}\text { Medically } \\
\text { related } \\
\text { absenteeism }\end{array}$ & $\$ 6,329$ & $(\$ 7,136)$ & $\$ 5,687$ & $(\$ 6,200)$ & 0.5012 \\
\hline Disability & $\$ 4,970$ & $(\$ 10,994)$ & $\$ 3,356$ & $(\$ 10,284)$ & 0.3134 \\
\hline
\end{tabular}

SD indicates standard deviation.

${ }^{a} P$-values are determined using McNemar tests for proportions and paired $t$-tests for continuous measures.

${ }^{b}$ Workloss rate defined as the number of people with $\geq 1$ disability claim or $\geq 1$ instance of medically-related absenteeism.
The results suggest that CAPN patients are associated with a significant and substantial economic burden among the privately insured U.S. population. Cases with CAPN had on average $\$ 17,344$ higher healthcare costs during the 12month study period compared with controls without CAPN. Compared with controls, more cases were hospitalized, had an emergency department visit, saw an oncologist or neurologist, and had other outpatient visits. In addition, each case with CAPN averaged 12 more outpatient visits and spent more days in the hospital. This suggests that in addition to the excess cost burden to third-party payors, the patients themselves may (depending on their insurance benefits) experience a large burden in terms of out-of-pocket costs (e.g., copayments, coinsurance) and time spent on medical care.

CAPN can lead to increased costs as a result of services specifically aimed at mitigating the PN (e.g., increased physician visits to monitor the $\mathrm{PN}$, costs of $\mathrm{PN}$ treatment) and secondary effects, such as switches in chemotherapy regimens or exacerbated cancer. This study separately estimated the costs for CAPN-related services and found they accounted for less than $2 \%$ of healthcare costs during the 12-month study period. While a significantly higher proportion of CAPN patients did use the pharmacologic treatments used to manage neuropathic symptoms, these drugs are often generic or lower cost relative to cancer treatments and overall healthcare costs. CAPN-related medical costs were low suggesting that the excess utilization demonstrated by cases was not directly attributed to CAPN. However, the CAPN-related costs as measured here may understate the true burden related specifically to CAPN. First, because there 
are no specific diagnoses for CAPN, PN-related diagnoses may be underreported on claims. In addition, the secondary effects of CAPN mentioned above are not included in the CAPN-related subset of costs presented here but rather are included in the chemotherapy, drug, and medical costs.

Overall, this study finds that patients with CAPN experience significantly increased costs and resource use. It is important not to conclude that all of the excess costs are caused by CAPN. Since the cases and controls were matched during the baseline period, the results suggest that CAPN is a serious condition that should be carefully monitored in clinical practice. Improvements to the diagnostic tools for CAPN severity and investigation of therapies that treat CAPN without negatively impacting the cancer treatment could benefit both patients and payors.

Calhoun's pilot study of chemotherapy-induced toxicity found that the direct CAPN costs for women with ovarian cancer are $\$ 688$ for episodes up to 9 months, compared with our findings that CAPN-related costs are on average $\$ 1,320$ over a period up to 12 months [12]. Calhoun's study relied on patient reported utilization and standard fee data whereas data here are actual reimbursements, which may, in addition to the difference in length of followup, explain the differences in estimates. Calhoun also analyzed indirect costs based on national labor force, employment, and earnings data, including patient workloss, caregiver workloss, and paid caregiver costs. Calhoun found indirect costs were $\$ 4,220$, with $67 \%$ of that due to caregiver workloss. Patient workloss costs were $\$ 620$ per patient per episode compared with our findings of $\$ 642$ per patient per year in excess medically related absenteeism costs. Though Calhoun did not include disability costs, this study found CAPN patients had mean excess per patient per year disability costs of $\$ 1,614$. While the workloss findings were not statistically significant, this may be due to the small sample size. It is important to note that the workloss cost estimates reported in this study may differ from actual employer costs depending on the employer's paid time off policies.

Healthcare costs and resource use were calculated for diabetic CAPN patients as a secondary analysis as literature suggests that diabetes may exacerbate the risk and severity of CAPN. With the prevalence of diabetes increasing, it is important to assess how diabetic CAPN patients may differ from controls and nondiabetic CAPN patients. The results demonstrated that diabetes may, indeed, be associated with increased cost in patients with CAPN, as excess costs of diabetic CAPN patients were $25 \%$ higher than for nondiabetic CAPN patients. However, the comparison between diabetic and nondiabetic CAPN patients was not statistically significant, perhaps due to the small sample of diabetic CAPN patients. In addition, although diabetic cases were balanced with their controls in terms of costs and resource use, a higher proportion of cases had metastatic cancer and the CCI of cases was significantly higher than controls. Both these factors could impact the excess cost findings.

This study is limited by the lack of clinical measures, which is common to research that uses claims data. While propensity score matching was used to adjust for many baseline group differences, the possibility exists of other confounding factors not available in this database. Moreover, because CAPN does not have a specific diagnosis code and is not consistently recorded in claims data, there are challenges in identifying the condition. Physicians may not report CAPN unless the impairment is severe enough to affect a patient's activities of daily living or warrant alterations to cancer treatment [1]. Thus, this study may include more severe cases of CAPN and may have under identified CAPN patients. The algorithm used for identifying CAPN in this study has not been validated. It is possible that the PN identified was due to other causes. Another study limitation is that due to data availability, cases and controls are matched using only 3 months of baseline data and information on cancer stage beyond metastatic/nonmetastatic is not available. A longer baseline period using a larger database with clinical information on cancer stage may allow for additional controls. The lack of restrictions on the length of continuous eligibility following index date is another possible limitation. This limitation could potentially lead to censoring of costs if a patient's plan were to have withdrawn from the database while the patient was continuing to incur costs; however, no such instances occurred in the study and the average length of followup for patients in the study is 11.1 months. Finally, small sample sizes, particularly in the head and neck, ovarian, workloss cost, and diabetic analyses, limited both the types of comparisons as well as the robustness of the statistical inferences. Though this study reports directional differences in workloss outcome measures, no significant differences were found, potentially due to the small sample size.

\section{Conclusion}

CAPN patients are associated with significantly higher healthcare costs and resource use in patients with breast, ovarian, head/neck, or NSCLC. The excess healthcare cost of CAPN is underestimated when only the cost for medical or pharmacy claims directly for CAPN is considered. Improvements in clinical assessments and treatments for CAPN would be useful for patients and payors.

\section{Acknowledgments}

R. B. Natale of Cedars-Sinai Medical Center, Los Angeles, CA, USA served as a clinical consultant to the study. The authors would like to thank Rebecca Kaufman for her assistance with this study. Previous presentations of this study are as follows: C. T. Pike, H. G. Birnbaum, R. J. Kaufman, C. E. Muehlenbein, G. M. Pohl, R. B. Natale, direct healthcare and workloss burden of chemotherapyassociated peripheral neuropathy in breast, ovarian, head and neck, and nonsmall cell lung cancer; value Health 2009; 12(3):A1-A19. Oral Presentation ND2 at ISPOR 14th Annual International Meeting, 16-20 May 2009, Orlando, Florida, United States. This study was sponsored by Eli Lilly and Company, Indianapolis, IN, USA. C. Muehlenbein and G. M. Pohl are employees of Eli Lilly and Company. C. T. Pike and H. G. Birnbaum are employees of Analysis Group, Inc., Boston, MA, which received funding for this study. 


\section{References}

[1] F. H. Hausheer, R. L. Schilsky, S. Bain, E. J. Berghorn, and F. Lieberman, "Diagnosis, management, and evaluation of chemotherapy-induced peripheral neuropathy," Seminars in Oncology, vol. 33, no. 1, pp. 15-49, 2006.

[2] S. Quasthoff and H. P. Hartung, "Chemotherapy-induced peripheral neuropathy," Journal of Neurology, vol. 249, no. 1, pp. 9-17, 2002.

[3] J. J. Lee and S. M. Swain, "Peripheral neuropathy induced by microtubule-stabilizing agents," Journal of Clinical Oncology, vol. 24, no. 10, pp. 1633-1642, 2006.

[4] H. Gogas, F. Shapiro, C. Aghajanian et al., "The impact of diabetes mellitus on the toxicity of therapy for advanced ovarian cancer," Gynecologic Oncology, vol. 61, no. 1, pp. 2226, 1996.

[5] C. C. P. Verstappen, J. J. Heimans, K. Hoekman, and T. J. Postma, "Neurotoxic complications of chemotherapy in patients with cancer: clinical signs and optimal management," Drugs, vol. 63, no. 15, pp. 1549-1563, 2003.

[6] B. Dunlap and J. A. Paice, "Chemotherapy-induces peripheral neuropathy: a need for standardization in measurement," Journal of Supportive Oncology, vol. 4, no. 8, pp. 398-399, 2006.

[7] M. A. Wampler and E. H. Rosenbaum, "Chemotherapyinduced peripheral neuropathy fact sheet. Cancer Supportive Care Programs," 2008, http://www.cancersupportivecare .com/nervepain.php.

[8] S. Mielke, A. Sparreboom, and K. Mross, "Peripheral neuropathy: a persisting challenge in paclitaxel-based regimes," European Journal of Cancer, vol. 42, no. 1, pp. 24-30, 2006.

[9] T. J. Kaley and L. M. Deangelis, "Therapy of chemotherapyinduced peripheral neuropathy," British Journal of Haematology, vol. 145, no. 1, pp. 3-14, 2009.

[10] M. M. Backonja, "Use of anticonvulsants for treatment of neuropathic pain,” Neurology, vol. 59, no. 5, pp. S14-S17, 2002.

[11] A. Berger, E. M. Dukes, and G. Oster, "Clinical characteristics and economic costs of patients with painful neuropathic disorders," Journal of Pain, vol. 5, no. 3, pp. 143-149, 2004.

[12] E. A. Calhoun, C. H. Chang, E. E. Welshman, D. A. Fishman, J. R. Lurain, and C. L. Bennett, "Evaluating the total costs of chemotherapy-induced toxicity: results from a pilot study with ovarian cancer patients," Oncologist, vol. 6, no. 5, pp. 441445, 2001.

[13] P. R. Rosenbaum, "Optimal matching for observational studies," Journal of the American Statistical Association, vol. 84, pp. 1024-1032, 1989.

[14] M. E. Charlson, P. Pompei, K. A. Ales, and C. R. MacKenzie, "A new method of classifying prognostic comorbidity in longitudinal studies: development and validation," Journal of Chronic Diseases, vol. 40, no. 5, pp. 373-383, 1987. 


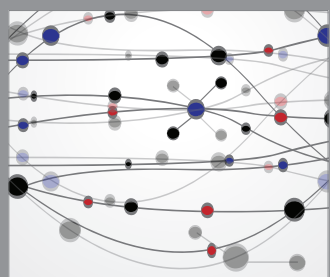

The Scientific World Journal
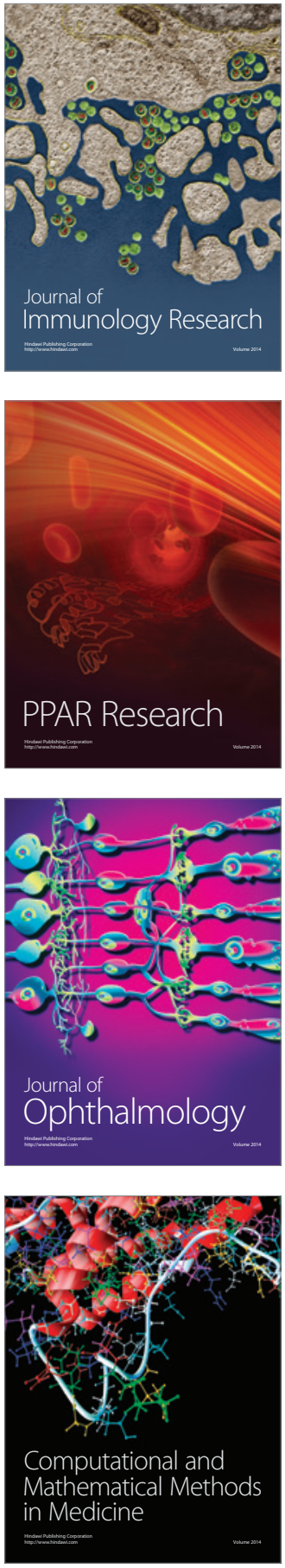

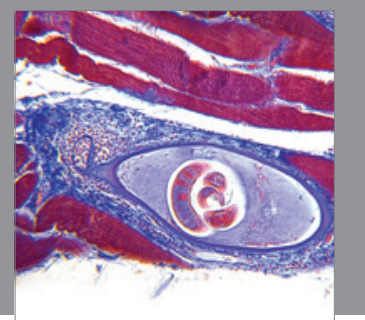

Gastroenterology

Research and Practice
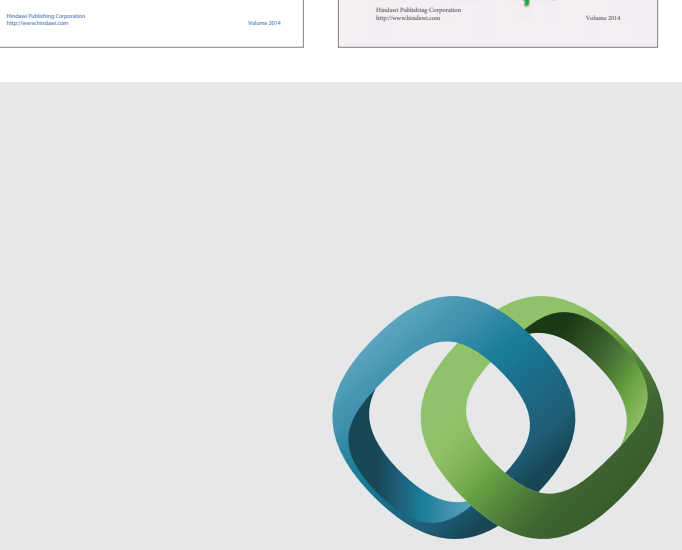

\section{Hindawi}

Submit your manuscripts at

http://www.hindawi.com
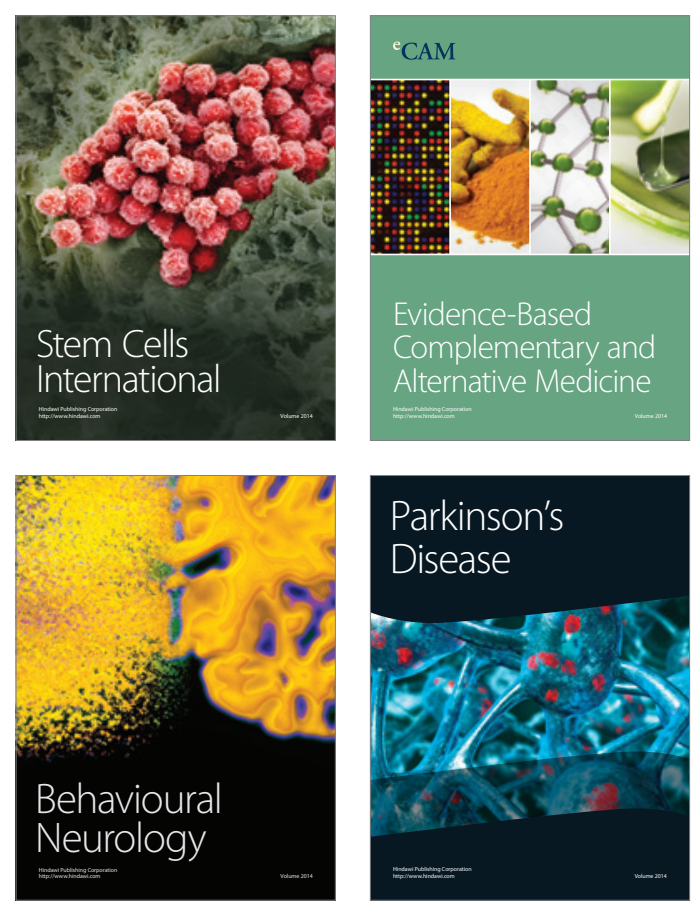

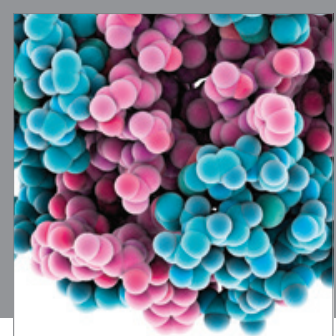

Journal of
Diabetes Research

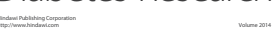

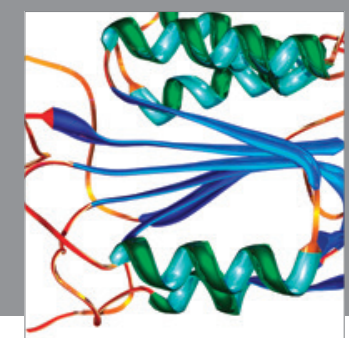

Disease Markers
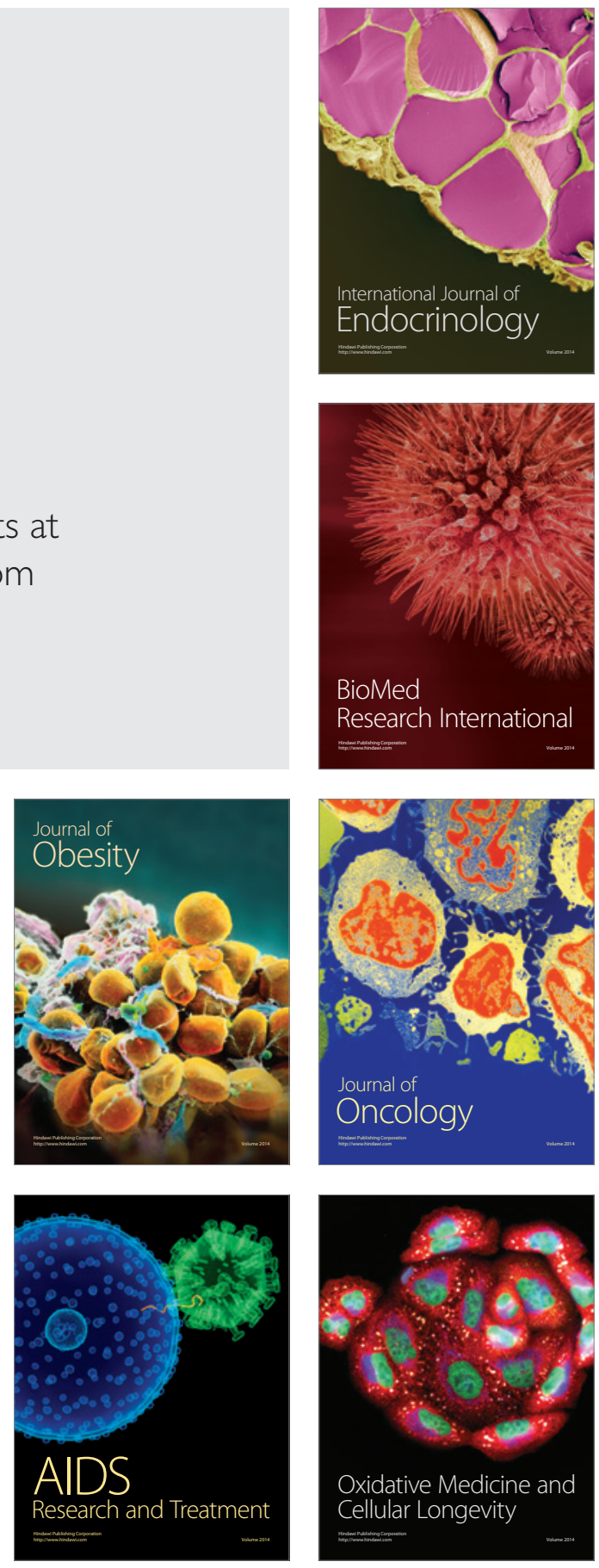